\title{
A COMMUTATOR ESTIMATE FOR PSEUDO-DIFFERENTIAL OPERATORS
}

\author{
JÜRGEN MARSCHALL
}

(Communicated by Walter D. Littman)

\begin{abstract}
For the commutator $B \cdot A-\mathrm{Op}(b a)$ of two pseudo-differential operators $A$ and $B$ an estimate on weighted Sobolev spaces is proved under minimal regularity assumptions on the symbols $a$ and $b$.
\end{abstract}

1. Let $a, b: \mathbf{R}^{n} \times \mathbf{R}^{n} \rightarrow \mathbf{C}$ two symbols. We impose the following conditions on $a$ and $b$. Let $N$ be a natural number and suppose that for all multi-indices $\alpha$ such that $|\alpha| \leq N$ there holds

$$
\begin{aligned}
& \left|D_{\xi}^{\alpha} b(x, \xi)\right| \leq C(1+|\xi|)^{-|\alpha|}, \\
& \left|D_{x_{i}} D_{\xi}^{\alpha} b(x, \xi)\right| \leq C * \Omega(|\xi|)(1+|\xi|)^{-|\alpha|}
\end{aligned}
$$

if $i=1, \ldots, n$. Suppose further that for all $\alpha$ such that $|\alpha| \leq n$ there holds

$$
\begin{aligned}
& \left|D_{\xi}^{\alpha} a(x, \xi)\right| \leq C(1+|\xi|)^{-|\alpha|}, \\
& \left|D_{x_{i}} D_{\xi}^{\alpha} a(x, \xi)\right| \leq C(1+|\xi|)^{-|\alpha|} \\
& \left|D_{x_{i}} D_{\xi}^{\alpha}(a(x+h, \xi)-a(x, \xi))\right| \leq C \omega(|h|,|\xi|)(1+|\xi|)^{-|\alpha|} .
\end{aligned}
$$

We suppose that for each $t>0$ the function $\omega(\cdot, t)$ is increasing and concave and that the functions $\omega(t, \cdot)$ and $\Omega$ are almost increasing in the following sense. There exists a positive constant $C$ independent of $t$ such that

$$
\omega(t, \tau) \leq C \omega(t, s)
$$

whenever $0.5 * \tau \leq s \leq 2 * \tau$ and similarly for $\Omega$.

2. Let $w$ be a positive, locally integrable function. We say that $w \in A_{p}$, i.e. $w$ satisfies Muckenhoupt's $A_{p}$-condition for some $1<p<\infty$, iff

$$
\sup \frac{1}{|Q|} \int_{Q} w d x\left(\frac{1}{|Q|} \int_{Q} w^{-1 /(p-1)} d x\right)^{p-1}<\infty
$$

where the supremum is taken over all cubes $Q \subset \mathbf{R}^{n}$. Denote by $L^{p}(w)$ the weighted $L^{p}$-space and let $J^{s}$ be the Bessel potential of order $s \in \mathbf{R}$. The weighted Sobolev space $H^{s, p}(w)$ is defined to be the space of all tempered distributions $f$ such that

$$
\|f\|_{H^{s, p}(w)}:=\left\|J^{-s} f\right\|_{L^{p}(w)}<\infty
$$

(compare Miler [7]).

Received by the editors April 10, 1987.

1980 Mathematics Subject Classification (1985 Revision). Primary 35S05, 47G05.

Key words and phrases. Pseudo-differential operators, commutators, weighted Sobolev spaces. 
3. Our main objective is to prove the following result.

THEOREM 1. Let $N=n+[n / 2]+2$ and suppose that the symbols $a$ and $b$ satisfy (1), (2) and (3), (4), (5) respectively. Let $\omega$ and $\Omega$ satisfy (6) and be such that $\left\{2^{-j} \Omega\left(2^{j}\right)\right\},\left\{\omega\left(2^{-j}, 2^{j}\right)\right\} \in l^{2}(\mathbf{N})$. Suppose that $1<p<\infty, w \in A_{p}$ and $0 \leq s \leq 1$. Then the commutator

$$
B \cdot A-\mathrm{Op}(b a): H^{s-1, p}(w) \rightarrow H^{s, p}(w)
$$

is bounded.

This theorem extends earlier results by Kumano Go and Nagase [3] and Bourdaud [1]; see also Marschall [4].

4. Before we prove the theorem let us provide a result needed in the proof. Let $c: \mathbf{R}^{n} \times \mathbf{R}^{n} \rightarrow \mathbf{C}$ be a symbol and suppose that for all multi-indices $\alpha$ such that $|\alpha| \leq n$ there holds

$$
\begin{aligned}
& \left|D_{\xi}^{\alpha} c(x, \xi)\right| \leq C(1+|\xi|)^{-|\alpha|} \\
& \left|D_{\xi}^{\alpha}(c(x+h, \xi)-c(x, \xi))\right| \leq C \tilde{\omega}(|h|,|\xi|)(1+|\xi|)^{-|\alpha|} .
\end{aligned}
$$

Proposition 2. Let $1<p<\infty$ and $w \in A_{p}$.

(i) If the symbol c satisfies (9) and (10) with a function $\tilde{\omega}$ such that (6) and $\left\{\tilde{\omega}\left(2^{-j}, 2^{j}\right)\right\} \in l^{2}(\mathbf{N})$ hold, then the operator $C: L^{p}(w) \rightarrow L^{p}(w)$ is bounded.

(ii) Suppose that (9) holds and that for each $\xi \in \mathbf{R}^{n}$ the function $c(\cdot, \xi)$ has its spectrum contained in the ball $\left\{\eta:|\eta| \leq 0.1 *\left(1+|\xi|^{2}\right)^{1 / 2}\right\}$. Then for every real number $s$ the operator $C: H^{s, p}(w) \rightarrow H^{s, p}(w)$ is bounded.

For a proof of the proposition see Marschall [5] and also Coifman and Meyer [2].

5. We are now in the position for the

PROOF OF THE THEOREM.

Step (i). Let $K$ be a function belonging to the Schwartz space $S\left(\mathbf{R}^{n}\right)$ such that the spectrum of $K$ is contained in the ball $B(0,0.05)$ and that the Fourier transform of $K$ is equal to one in a neighborhood of the origin. Define

$$
K_{\xi}(x):=\left(1+|\xi|^{2}\right)^{n / 2} K\left(\left(1+|\xi|^{2}\right)^{1 / 2} x\right)
$$

and decompose the symbols $a$ and $b$ as follows. Let

$$
\begin{aligned}
& a_{1}(x, \xi):=\int K_{\xi}(y) a(x-y, \xi) d y \\
& b_{1}(x, \xi):=\int K_{\xi}(y) b(x-y, \xi) d y
\end{aligned}
$$

and $a_{2}:=a-a_{1}$ and $b_{2}:=b-b_{1}$. Note that by the conditions on the Fourier transform of $K$ one has

$$
\int K_{\xi}(y) d y=1, \quad \int y_{i} K_{\xi}(y) d y=0, \quad i=1, \ldots, n .
$$

Hence, it follows that

$$
\begin{aligned}
& \left|D_{\xi}^{\alpha} b_{2}(x, \xi)\right| \leq C * \Omega(|\xi|)(1+|\xi|)^{-1-|\alpha|} \\
& \left|D_{x_{i}} D_{\xi}^{\alpha} b_{2}(x, \xi)\right| \leq C * \Omega(|\xi|)(1+|\xi|)^{-|\alpha|} \\
& \left|D_{\xi}^{\alpha} a_{2}(x, \xi)\right| \leq C \omega\left((1+|\xi|)^{-1},|\xi|\right)(1+|\xi|)^{-1-|\alpha|} \\
& \left|D_{x_{i}} D_{\xi}^{\alpha} a_{2}(x, \xi)\right| \leq C \omega\left((1+|\xi|)^{-1},|\xi|\right)(1+|\xi|)^{-|\alpha|} .
\end{aligned}
$$


For example, in order to prove (13) observe that

$$
a_{2}(x, \xi)=\int K_{\xi}(y)\left\langle a(x, \xi)-a(x-y, \xi)-\sum_{i=1}^{n} y_{i} \frac{\partial a}{\partial x_{i}}(x, \xi)\right\rangle d y
$$

and hence, by the mean value theorem

$$
\begin{aligned}
\left|a_{2}(x, \xi)\right| & \leq C \int|y|\left|K_{\xi}(y)\right| \omega(|y|,|\xi|) d y \\
& \leq C(1+|\xi|)^{-1} \int|y||K(y)| \omega\left((1+|\xi|)^{-1}|y|,|\xi|\right) d y .
\end{aligned}
$$

Now, $\omega(\cdot,|\xi|)$ being increasing and concave, one has

$$
\omega\left((1+|\xi|)^{-1}|y|,|\xi|\right) \leq(1+|y|) \omega\left((1+|\xi|)^{-1},|\xi|\right)
$$

(compare Coifman and Meyer [2]) and (13) follows.

Step (ii). By complex interpolation it suffices to prove the theorem in the endpoint cases $s=0$ and $s=1$. Consider first the term $C_{i}:=B_{i} \cdot A_{1}-\operatorname{Op}\left(b_{i} a_{1}\right)$, $i=1,2$. It has the symbol

$$
c_{i}(x, \xi)=\frac{1}{(2 \pi)^{n}} \sum_{j=1}^{n} \int_{0}^{1} \int \frac{\partial b_{i}}{\partial \xi_{j}}(x, \xi+t \eta)\left(D_{x_{j}} a_{1}\right)^{\wedge}(\eta, \xi) d \eta d t
$$

where $\left(D_{x_{j}} a_{1}\right)^{\wedge}(\cdot, \xi)$ is meant to be the Fourier transform of the function $D_{x_{j}} a_{1}(\cdot, \xi)$. Then, using a method by Meyer [6], it follows that for all multi-indices $\alpha$ such that $|\alpha| \leq n$ one has

$$
\begin{aligned}
& \left|D_{\xi}^{\alpha} c_{1}(x, \xi)\right| \leq C(1+|\xi|)^{-|\alpha|} \\
& \left|D_{\xi}^{\alpha} c_{2}(x, \xi)\right| \leq C * \Omega(|\xi|)(1+|\xi|)^{-2-|\alpha|} \\
& \left|D_{x_{j}} D_{\xi}^{\alpha} c_{2}(x, \xi)\right| \leq C * \Omega(|\xi|)(1+|\xi|)^{-1-|\alpha|}
\end{aligned}
$$

But then the proposition and the condition $\left\{2^{-j} \Omega\left(2^{j}\right)\right\} \in l^{2}(\mathbf{N})$ yield the boundedness of

$$
C_{i}: H^{s-1, p}(w) \rightarrow H^{s, p}(w)
$$

Step (iii). Observe that part (i) of the proposition and the condition $\left\{2^{-j} \Omega\left(2^{j}\right)\right\}$ $\in l^{2}(\mathbf{N})$ imply

$$
\begin{aligned}
& B: L^{p}(w) \rightarrow L^{p}(w), \\
& B: H^{1, p}(w) \rightarrow H^{1, p}(w) .
\end{aligned}
$$

Further, the proposition and (13), (14) yield

$$
\begin{aligned}
& A_{2}: H^{-1, p}(w) \rightarrow L^{p}(w), \\
& A_{2}: L^{p}(w) \rightarrow H^{1, p}(w)
\end{aligned}
$$

and consequently we get the boundedness of the term $B \cdot A_{2}$. Since the remaining term $\mathrm{Op}\left(b a_{2}\right)$ is treated similarly, the theorem is proved completely.

6. Let us remark that one can introduce symbols $b$ with $N$ not an integer. Then using complex interpolation of symbols one can see that the theorem holds when $N=3 n / 2+1$. For such techniques we refer to Marschall [4]. 


\section{REFERENCES}

1. G. Bourdaud, Régularité du commutateur de deux opérateurs pseudo-différentiels peu réguliers, C. R. Acad. Sci. Paris Sér. A 290 (1980), 67-70.

2. R. R. Coifman and Y. Meyer, Au délà des opérateurs pseudo-différentiels, Astérisque 57 (1978), 1-185.

3. H. Kumano Go and M. Nagase, Pseudo-differential operators with nonregular symbols and applications, Funkcial. Ekvac. 21 (1978), 151-192.

4. J. Marschall, Pseudo-differential operators with nonregular symbols of the class $S_{\rho, \delta}^{m}$, Comm. Partial Differential Equations 12 (1987), 321-365.

5. __ Weighted parabolic Triebel spaces of product type, Fourier multipliers and pseudo-differential operators, Preprint.

6. Y. Meyer, Remarques sur un théorème de J. M. Bony, Rend. Circ. Mat. Palermo 2 (1981), Suppl. 1, 1-20.

7. N. Miller, Weighted Sobolev spaces and pseudo-differential operators with smooth symbols, Trans. Amer. Math. Soc. 269 (1982), 91-109.

FAKULTÄT FÜR INFORMATIK, UNIVERSITÄT DER BUNDESWEHR MÜNCHEN, WERNERHEISENBERG-WEG 39, D-8014 NEUBIBERG, FEDERAL REPUbLIC OF GERMANY 